\title{
Arquitectura Colaborativa. Una Aproximación al Tema a Través de 4 Casos
}

\author{
Collaborative Architecture. An Approach to the Topic Through \\ 4 Cases
}

\section{Arquitetura Colaborativa. Uma Abordagem para o Problema Através 4 Casos}

\author{
Javier Samaniego * \\ Universidad Nacional de La Plata
}

\begin{abstract}
En este escrito propongo aportar a la generación de enfoques alternativos sobre la arquitectura como práctica pedagógica en ámbitos extradisciplinares. A partir de mi experiencia como educador popular, analizaré el desplazamiento de la arquitectura en dirección a las artes, para el abordaje de problemáticas sociales y territoriales. Para ello, tomaré 4 casos de talleres destinados a niños, adolescentes y comunidades vulnerables y señalaré algunos aspectos relevantes para esbozar un cuerpo de ideas al que llamaré Arquitectura Colaborativa. Este último concepto es entendido como una producción relacional entre la arquitectura y el arte con la comunidad. Una serie de modelos de intercambio e implicación participativa capaces de dar nuevas formas discursivas en torno a la arquitectura. Transformando el rol de la comunidad en la producción del espacio, con el potencial de replicarse a otras realidades. En la primera parte definiré algunas ideas rectoras, en la segunda expondré lo casos, en la tercera y última arribaré a algunas conclusiones.
\end{abstract}

Descriptores: Arquitectura, Arte, Espacio, Comunidad, Participación.

In this paper, I propose to contribute to the generation of alternative approaches on architecture as pedagogical practice in interdisciplinary domains. From my experience as a popular educator, I will analyze the displacement of architecture toward the arts, at the service of certain social problems. To do this, I will take 4 cases of workshops designated to children, adolescents and vulnerable communities and I will point out some relevant aspects to outline a body of ideas that I will call Collaborative Architecture. Understood this as a Relational production between architecture and art with the community. A series of models of exchange and participative involvement capable of giving new discursive forms of architecture. Transforming the role of the community in the production of space, with the potential to replicate to other realities. In the first part I will define some guiding ideas, in the second part I will explain the cases, in the third and last I will arrive to some conclusions.

Keywords: Architecture, Art, Space, Community, Participation.

\footnotetext{
*Contacto: javiersamaniegog@gmail.com
}

ISSN: 2254-3139

www.rinace.net/riejs/

revistas.uam.es/riejs
Recibido: $\quad 21$ de diciembre 2016

$1^{\text {a }}$ Evaluación: 17 de enero 2017

$2^{\text {a }}$ Evaluación: 1 de abril 2017

Aceptado: $\quad 25$ de abril 2017 


\begin{abstract}
Neste artigo proponho a contribuir à geração de abordagens alternativos a arquitetura como prática pedagógica em áreas extra disciplinares. Da minha experiência como educador popular, vou analisar a deslocação da arquitetura em direção às artes, para a abordagem os problemas sociais e territoriais. Para fazer isso, tomarei 4 casos de oficinas para crianças, adolescentes e comunidades vulneráveis e assinalarei alguns aspectos relevantes para delinear um corpo de ideias ao que chamam Arquitetura Colaborativa. Este último conceito é entendido como uma produção relacional entre arquitetura e a arte com a comunidade. Uma série de modelos de intercâmbio e envolvimento participativo capazes de dar novas formas discursivas em torno da arquitetura. A transformação o papel da comunidade na produção do espaço, com o potencial para ser replicado a outras realidades. Na primeira parte definirei algumas ideias orientadoras, na segunda exporei o caso, na terça e última arribaré a algumas conclusões.
\end{abstract}

Palavras-chave: Arquitetura, Arte, Espaço, Comunidade, Participação.

\title{
La Arquitectura no es solo cosa de arquitectos
}

La arquitectura es un cuerpo de ideas, metodologías y prácticas que se proponen abordar y dar respuestas al problema del hábitat humano. Es considerada desde múltiples perspectivas y escuelas de pensamiento. Algunas se centran en el estudio del objeto arquitectónico, poniendo el foco en sus características físicas, espaciales, estéticas y materiales. Produciendo distintas valoraciones, categorizaciones y catalogaciones. Otros a su vez, la ven como una mercancía sujeta al intercambio y sometida al juego inmobiliario y marketinero de moda. Otros, en cambio, la entienden como un proceso dialéctico en el marco lucha de clases sociales. Reflejo de procesos políticos, económicos y culturales. Si bien existen posicionamientos enfrentados como resultado de disputas ideológicas, el punto está en que la arquitectura estuvo y está mostrada únicamente desde el ojo de la disciplina. Nadie duda de que el aprendizaje sobre espacio sea un tema que le compete a cualquier sujeto, sin embargo, la arquitectura termina siendo un tema solo de arquitectos. La sociedad, acostumbrada a verla desde el ojo monopólico de la disciplina, la mira con lejanía. A contrapelo, artistas, arquitectos, colectivos y organizaciones plantean un abordaje contra-hegemónico desde las artes colaborativas, la militancia territorial, la educación popular y la educación por el arte. Acercando la arquitectura al resto de la comunidad. Partiendo de este enfoque propongo una forma de encarar el tema a partir de mi experiencia docente y como artista en espacios extradisciplinares. El objetivo es contribuir al acceso libre a la formación en temas arquitectónicos, urbanos y artísticos para el resto de la comunidad no-disciplinar. Con esto logro formalizar un testimonio de la arquitectura surgido en la informalidad.

A continuación, expongo algunos lineamientos conceptuales y los 4 casos mencionados a fin de abrir el tema y delinear algunas pautas para seguir pensando y replicar en otros lugares. Con este artículo no busco develar algo que no se haya practicado/pensado, pero si identificar algunas coincidencias desde donde hilvanar un enfoque expandido de la arquitectura. 


\title{
1. Fundamentación teórica
}

\section{Lo colaborativo, lo transdisciplinar, lo híbrido y lo relacional}

En los últimos tiempos las problemáticas de la arquitectura han encontrado interlocución en el campo de algunas experimentaciones artísticas. Quisiera señalar el rol específico de algunas prácticas artísticas que, en el actual panorama, se afirman en el centro de los conflictos en los que focalicé mi atención sobre este tema. Es significativo entonces mencionar las artes colaborativas. Me interesa señalar los diferentes grados de implicación que existen entre artistas y la comunidad. Con la intención producir prácticas sobre el espacio y el territorio a comunidades específicas. Numerosos trabajos han abordado experiencias colaborativas, entre ellas el trabajo de Garrido (2009), Del Río Almagro y Collados Alcalde (2013), Claramonte (2012). Todos sostienen que, si bien existen tipos de implicación y coautoría en propuestas no colaborativas, en estas prácticas, se posibilitan nuevas formas discursivas de subjetividad e identidad. Las prácticas resultantes conciben al espacio como un sistema complejo productor de relaciones sociales capaces de construir marcos de trabajo cooperativos y organizaciones diversas constituyendo un campo hibridizado entre el arte, la política y la organización comunitaria. Del Río Almagro y Collados Alcaide (2013) dicen:

\begin{abstract}
En las prácticas artísticas colaborativas, tanto artistas como comunidad, se desprenden nuevos modos de hacer, nuevos modos de relación, generando formas insólitas de sociabilidad, modelos de mediación, participación y formas heterogéneas de asociación e interacción. La capacidad de establecer redes de trabajo, de instaurar vínculos, complicidades y de explorar nuevas formas operativas, en las que el tejido social quede implicado, como parte integrante del proceso artístico. (p. 17)
\end{abstract}

Otro rasgo que resulta interesante para pensar la arquitectura es su dimensión relacional. Como horizonte teórico para pensar una obra no como hecho físico espacial, sino como un proceso el marco de un universo de relaciones sociales que se despliegan y se nutren del contexto de la arquitectura. Una obra entendida desde un punto de vista relacional se extiende más allá de su forma material/física/espacial y se transforma en posibilidad de aglutinación. Bourriaud (2007) dice además que los artistas proponen como obras de arte: 1. momentos de lo social, 2. objetos productores de lo social. No utiliza un marco relacional definido a priori para obtener principios de producción. En este sentido, Bourriaud dice que el arte está hecho "de la misma materia que los intercambios sociales” (p. 49) y ocupa un lugar específico en la producción cultural. Supera su simple presencia en el espacio; se abre al diálogo, al intercambio y a la transformación.

Así, la arquitectura llevada a lo colaborativo y lo relacional convoca al intercambio desde la perspectiva centrada en la transversalidad de los saberes sobre el espacio. Se busca lo aglutinador de la arquitectura y la construcción de relatos situados. Con la posibilidad de producir intercambios en los casos que amerite. Fortaleciendo conocimientos y habilidades mediante experiencias simples en vinculación con el espacio cotidiano.

La obra arquitectónica no solo es concebida como un objeto físico, sino como una experiencia estética y ética en el marco de tensiones sociales y políticas. En este sentido se considera a la sociedad como su motor. En ella existe una fuerza potencial capaz de gestar respuestas locales creativas y construir otros modos particulares de observación, discusión, representación del espacio arquitectónico y urbano de una manera cabal. Sobre eso García Canclini (1999) dice: "las ciudades no son sólo un fenómeno físico, un 
modo de ocupar el espacio, de aglomerarse, sino también lugares donde ocurren fenómenos expresivos que entran en tensión” (p.72). De esta forma, la negociación y el conflicto son constituyentes en este proceso, así como también los modos de practicar y significar al espacio. En este espacio (privado y público) se producen entonces instancias de enunciación, las cuales pueden interpelar al orden de cosas hegemónico

\section{Métodos y resultados}

Este análisis sintetiza los últimos 10años de trabajo como docente de Arte y Arquitectura en ámbitos no disciplinares: en el Hogar de Niños Rayuela; la Escuela de Ciegos $\mathrm{N}^{\circ}$ 515; el Consejo de Integración para personas con Discapacidad de La Plata y el Centro de Asistencia Familiar de la Villa 31 del barrio de Retiro de la Ciudad Autónoma de Buenos Aires.

En cada caso hubo una constante revisión y ajuste de estrategias pedagógicas de acuerdo a las diversas problemáticas. Las consignas de trabajo son flexibles y genéricas admitiendo particularidades y emergentes que surgieron en su desarrollo.

\subsection{Caso 1: Pequeños arquitectos}

Hogar de Niños Rayuela, La Plata (Buenos Aires).

Duración de 2007/2015

Centro de día de Rayuela. Es una ONG que tiene como destinatarios a Niños con causas asistenciales. El mismo tiene una doble modalidad: 1) Hogar convivencial: residen niños con causas asistenciales bajo la tutela de un Juez de Menores. 2) Centro de día: asistencia diaria a niños y familias residentes en los alrededores. Desde la Institución se aborda la situación integral del niño trabajando líneas de acción de acuerdo al caso y la problemática.

El taller Pequeños arquitectos surge como iniciativa propia a la institución para trabajar con los niños que la integran y se planteó, en sus inicios, como actividad recreativa a partir de una serie de encuentros lúdicos, salidas, meriendas, festejos, etc. El grupo compuesto por 12 niños de entre 6 y 13 años, de los cuales muchos de ellos son hermanos.

\section{Metodología}

El taller se estructura a partir de módulos.

- Percepción espacial: A partir de diferentes disparadores, proceso de experimentación sobre la cualidad del espacio cotidiano: la escuela, la plaza, la casa. Registros. Los tangible y lo intangible. Percepción espacial

- Abstracción espacial: Reconocimiento del espacio como construcción mental. Imágenes mentales espaciales. Los sueños, las fantasías. La escala mental y la escala humana. Memoria sensorial. Iconografías, símbolos espaciales.

- Cartografías arquitectónicas/urbanas: Juego de escalas. Lo gigante y la miniatura. Construcción de dispositivos cartográfico. Juegos con uso de mapa. Recorridas mapeadas. 
- Espacio de reflexión infantil: Charlas, Cines debate, Documentales. Usos y obstáculos.

Estas consignas retoman algún tema específico de la Arquitectura cruzado con temas de interés de los niños. Las mismas paulatinamente incorporaron una mayor complejidad. $\mathrm{El}$ arte y su potencial hibridación de enfoques, lenguajes y técnicas de representación del espacio. A través de distintas actividades, se estudió el barrio y la ciudad. Se produjeron registros, propuestas, intervenciones en el barrio. Finalmente se armó una mesa de debate infantil. Se abordaron conflictos surgidos en la movilidad peatonal y el uso del espacio público.

\subsection{Caso 2: Ciudad invisible}

La Plata (Buenos Aires)

Duración: 2011/2012

Equipo: Director Arq. Javier Samaniego García; Codirector Mg. Arq Laura Aon; Becarios: Federico Ortiz (FAU-UnLP) y Debora Fulco (FAU-UnLP), y Talleristas Docentes: Arq. Natalia Colantonio, Arq. Mariana Navarrete, Arq. Evangelina Fileni, Arq. Valeria Trapani, Celina Basualdo y Arq. Victoria Schiaffino (FAU-UnLP)

Identificación del/los destinatarios: Alumnos y Egresados de la Escuela $\mathrm{N}^{\circ} 515$ para Ciegos y Disminuidos Visuales, Gonnet, La Plata. Buenos Aires, Argentina. Su convocatoria incluye a los partidos de La Plata, Berisso, Ensenada, Brandsen y Berazategui. Asisten 200 alumnos ciegos y disminuidos visuales entre 3 y 18 años. Existe una gran diversidad en relación a las patologías visuales y derivadas. En esta institución se aprenden herramientas de autovalimiento: lectura y escritura en braille, educación física, manejo de bastón, etc.

Ciudad Invisible surge como una investigación acerca de la dimensión intangible de la arquitectura. Nace como un proyecto de un curso de posgrado de la Facultad de Arquitectura de la Universidad Nacional de La Plata. Compuesto por una serie de ideas entorno a la percepción no visible del espacio, como intento de aportar a la reflexión de los límites de la disciplina. $\mathrm{O}$, mejor dicho, la migración de la arquitectura a ámbitos y experiencias no-disciplinares. Con la intención producir obras de arquitectura en articulación con las artes engendrando un terreno inédito.

\section{Metodología}

Los objetivos metodológicos incluyen:

- Construir un plan de lectura cartográfica a partir del tacto, el sonido y el olfato para el análisis del espacio en distintas escalas (mental, humana, arquitectónica, barrial y urbana).

- Desarrollar metodologías de participación orientadas a identificar la percepción y representación invisible del espacio público.

- Mapear la movilidad, el uso del espacio público y el nivel de accesibilidad de la comunidad no-vidente. 
Fase 1:

- Invitación: Abierta a instituciones que abordan la problemática de la ceguera. Armado de sitio virtual de divulgación del proceso, participación, información, comunicación y debate.

- Encuadre y comunicación: Organización y encuadre con instituciones coparticipantes y Unidades académicas.

- Convocatoria: Para aspirantes a talleres de debate, utilizando canales de comunicación virtual, medios de comunicación.

- Taller de Debate: Debate de los temas significativos en el uso no-vidente de la ciudad.

- Trabajo de Campo: Recorridos urbanos exploratorios.

Fase 2:

- Taller diseño: Identificación áreas, focos, ejes, itinerarios del casco fundacional. Identificando su percepción a través de experiencias sensibles.

- Formulación de Propuestas: Espacios de recreación. Itinerarios olfativos, táctiles.

- Formación: Capacitación en Manejo de técnicas de comunicación y expresión para no videntes.

- Inclusión de la población no vidente en la participación Diseño Urbano Multisensorial (DUM).

- Trabajo de Campo: Itinerarios.

Fase 3

- Intervenciones: Murales, intervenciones y diseño de elementos de arte urbano audio táctil. Intervenciones urbanas, estables y efímeras (recorridos sensoriales y perceptivos)

- Evaluación y Monitoreo: Reuniones, supervisión de casos.

- Muestra de los resultados: Proyecto de inclusión y plan de actividades. Difusión de material en gacetilla impresa y en braille.

Se construyeron una serie de dispositivos espaciales multisensoriales y algunas consideraciones sobre la intangibilidad de la arquitectura. Con una doble intención, por un lado, para el señalamiento de las barreras arquitectónicas que atraviesa la población no-vidente y por el otro, para proveer la experiencia urbana por medio del olfato, el tacto y el audio.

Ciudad Invisible dio como resultado una vasta producción de obras visuales y audiovisuales. Proyectos habitacionales, equipamiento y espacio público, itinerarios urbanos temáticos. Dibujos, escrituras en braille, cartografías, maquetas, fotos y videos. Exposiciones y asistencias a encuentros destinados a debatir sobre temática de la educación por el arte, discapacidad y arquitectura. Con la intención de invitar a la participación directa de la población no-vidente en la toma de decisiones sobre la calidad 
táctil, sonora y olfativa de los espacios de la arquitectura y la Ciudad. Dar el lugar para que la percepción invisible se haga presente, generando el espacio y las condiciones para enunciarla.

\subsection{Caso 3: GAYA- Galería de Arte y Arquitectura}

Dirección de Integración para Personas con Discapacidad. La Plata (Buenos Aires).

Duración: de 2007-2017

Dirección de Integración. Es una institución que aborda la problemática de la discapacidad mental. Se dictan talleres laborales, educativos y artísticos. Atención psicológica, asistencia familiar y demás actividades recreativas y de integración. Las patologías mentales trabajadas son psicosis, esquizofrenia, autismo, retraso mental leve y moderado. Los integrantes de GAYA, provienen de distintos sectores de región de la ciudad de La Plata. En algunos casos, desarrollan una vida autónoma y otros necesitan ser acompañados por un familiar o acompañante terapéutico. En todos los casos, existen largas historias de institucionalización en lo referido a la salud, a la educación y la gestión de becas, pases de transporte, etc. Existe un total desconocimiento acerca de las posibilidades que un discapacitado tiene para desenvolverse. Las personas con discapacidad reciben un mensaje descalificador y una mirada compasiva. En muchos casos son conscientes de las injusticias y obstáculos que atraviesan cotidianamente.

El Taller GAYAes un espacio de reflexión y debate de Arquitectura. Su objetivo principal es construir un espacio especializado en formación y de divulgación sobre Arte contemporáneo y la Arquitectura para personas con discapacidad. En este sentido se trabaja con una lógica que articula cuerpos teóricos provenientes de distintas disciplinas y en solidaridad con las artes. Por medio de actividades y aproximaciones a la arquitectura con absoluta libertad de expresión, a tal punto de que no parecer a lo que comúnmente llamamos arquitectura. La producción de GAYApone el acento en aquellas producciones sobre la arquitectura que por incoherentes, incorrectas e impropias quedaron por fuera de lo que se llama "arquitectura". Sus obras además de testimoniar experiencias sensibles con espacio, son escrituras en sí misma y emancipadas del correlato proyectual. Posibilitan formas discursivas nuevas. Productoras de conocimiento a sobre en un marco de intercambios de diversidades sensibles.

Metodología

- Taller de Arquitectura: Taller de contenidos Arquitectónicos. Encuentros semanales. Proceso de 3 Fases. 1) Registro del espacio, recorridos, observación. 2) Desarrollo de temas. Investigación sobre la arquitectura contemporánea local platense. 3) Proyectos y propuestas. 4) obras colectivas-instalaciones artísticas.

- Galería: Lugar de exhibición permanente y transitoria.

- Colectivo de Arte: Proceso de construcción de una identidad colectiva. Manifiestos, escritos y acciones en el marco de un proceso de fortalecimiento del grupo como colectivo de arquitectura sin arquitectos.

- Trabajo Colaborativo: Convocatoria a artistas locales interesados en la Arquitectura. Valeria González (fotografía) Francisco Sendra (Teatro) Juan 
Castiglione, Gonzalo Cuadrado, Federico Ortiz (Audiovisual), Silvina Cavallaro (cerámica), y Leonardo Gauna (Artes Plásticas-Ilustración).

- Exhibiciones: De manera rotativa tomamos un artista, una disciplina artística y una obra de Arquitectura. Trabajamos un determinado período de investigación para luego hacer propuestas y exhibimos los resultados.

\subsection{Caso 4: Arquitectura barrial}

TURBA-Taller de Urbanismo Barrial, Villa 31 y 31 Bis. Buenos Aires.

Duración: 2009/2017

Equipo: Diego Danei, Julia Ramos, Pablo Vitale, y Javier Samaniego García.

TURBA es un colectivo artístico que se propone generar instancias participativas de reflexión sobre la arquitectura en las villas. Se aborda el espacio desde su escala barrial y una perspectiva social atravesada por una mirada comunitaria. Para ello se pone el foco en la relación que el espacio tiene con sus bases culturales en un marco de lucha política.

El Taller. Se desarrolla el taller desde el año 2009 destinado a jóvenes y adolescentes en la Villa 31/31 bis -emplazada en el barrio de Retiro de la Ciudad Autónoma de Buenos Aires- en donde se llevan adelante diversas actividades tendientes a visibilizar la existencia del barrio como parte de la ciudad y promover su apropiación por parte de la comunidad. La línea de trabajo preponderante es la producción de una cartografía popular que constituye una forma de representación del territorio a partir de una amplia batería de recursos artísticos, arquitectónicos y sociológicos. Se ha realizado un Mapa Abierto en donde converge toda la información relevada por el equipo de adolescentes participantes a partir de distintas estrategias. Se llama Abierto porque al igual que es territorio es susceptible de ser modificado y transformado conteniendo los aportes de cada persona o colectivo que desee participar. La idea de Mapa Abierto responde justamente a la apertura o permeabilidad respecto a las observaciones y apropiaciones de los usuarios/comunidad y a una perspectiva que concibe al espacio de naturaleza dinámica y en permanente construcción.

Para llegar a este Mapa Abierto, el proceso de trabajo se puede clasificar en tres etapas. Un primer momento de familiarización de los adolescentes con las distintas herramientas y conceptos urbanísticos y cartográficos a través de distintas actividades. En este marco se trabajó alrededor de dimensiones metodológicas y teóricas para la planificación de los barrios en sus diferentes escalas: cuadra, manzana, barrio, ciudad, regional, metropolitano, provincial, nacional. Un segundo momento de corte experimental en donde se exploraron aspectos ligados a la morfología y la representación urbana y un tercer bloque centrado en el relevamiento de información para la confección del mapa. Y un tercer momento de puesta a prueba en donde este mapa circuló por numerosas organizaciones, centros culturales y comunitarios, espacios educativos, etc., en donde se compartió el mapa y se realizó un taller a fin de ponerlo a revisión y nutrirlo de las opiniones, sugerencias, observaciones y aportes de las personas que participaron en cada encuentro. Con este fin se realizó un taller de urbanismo barrial a cargo del equipo de TURBA en cada espacio a fin de volcar los emergentes (sugerencias, correcciones, modificaciones y nueva información) en el mapa abierto para ir cobrando nuevas configuraciones conteniendo la subjetividad y la perspectiva espacial de los distintos actores participantes. 


\section{Metodología}

El taller se organizó a en 2 grandes momentos.

\section{Momento 1. Taller de Arquitectura y mapas}

- Fase 1. Diagnóstico y exploración de la escala barrial:

$\checkmark$ Conocimiento y familiarización con los conceptos y las técnicas de la elaboración de cartografías.

$\checkmark$ Identificación de los trayectos cotidianos. Usos, sensaciones, percepciones.

Relevamientos. Identificación de zonas importantes para la vida social y comunitaria de los habitantes del barrio (centros culturales, comunitarios, educativos, políticos, comedores, ferias, etc.), los puntos que dan sentido de pertenencia e identidad y finalmente la identificación de problemas en relación al hábitat que atraviesan los habitantes de la Villa.

\section{Trabajo final: mapeo colectivo}

- Fase 2. Escala urbana, de la sistematización y de la selección de ejes temático:

$\checkmark$ Actividades destinadas a explorar la escala urbana (reflexionando sobre los usos, trayectos y recorridos, las intersecciones con el barrio, los muros físicos y simbólicos existentes entre el barrio y la ciudad "formal", etc.)

Mapeos de la relación entre el barrio y su inserción urbana.

$\checkmark$ Selección de ejes temáticos

- Fase 3. Elaboración y sistematización de mapas:

$\checkmark$ Generación de imágenes, materiales audiovisuales y objetos que dieron cuenta de las distintas facetas abordadas por el proyecto.

$\checkmark$ Cartografías, materiales gráficos y audiovisuales.

Momento 2

- Mapeos itinerantes: Jornadas de mapeo y armado del Mapa Abierto. Sistematización y divulgación.

- Capacitación en Urbanismo Barrial, con el título de Cartógrafo Popular.

- Laboratorio Audiovisual Villero: Acciones artísticas, proyectos audiovisuales, talleres de formación en fotografía, cine y artes combinadas desde la villa para el resto de la ciudad.

- Mesa por la Urbanización infantil y adolescente: Encuentros de debate sobre la urbanización de las villas y el rol de los niños y adolescentes en el proceso de urbanización de Villas que se viene desarrollando.

La formación en arquitectura a los adolescentes de la villa posibilitó un reconocimiento y una valoración positiva por parte del resto de la comunidad al grupo adolescente. En 
este sentido, se identificaron de nuevas problemáticas espaciales y por el otro como un motor de diálogo para el reconocimiento de la población adolescente en la autogestión de la Villa. La práctica cartográfica es considerada como estrategia de formación de deseo (Rocha, 2014).

\section{Discusión y conclusiones}

En este escrito pretendo vincular cuatro prácticas educativas bajo el concepto de arquitectura colaborativa. Entendida esta como un concepto relacional, manifestado en prácticas educativas/artísticas al servicio de problemáticas socio-territoriales. En un marco que fluctúa entre la formalidad y la informalidad. Si bien las prácticas analizadas poseen problemáticas diferentes, pueden señalarse algunos puntos en común. En cada una de las experiencias comparadas existe una oscilación teórico-metodológica entre el arte colaborativo y la educación popular. Apoyados en una pedagogía que considera al sujeto como un factor clave para la orientación comunitaria. Motorizada por vínculos de compromiso, correspondencia y ayuda mutua.

Todos los casos han sido fructíferos en varios aspectos. Se logró una inserción al interior de cada institución, así como entre las instituciones de la comunidad. Asimismo, ha establecidos lazos de colaboración con colectivos, artistas e instituciones con temas afines produciendo intercambios, aportes y acciones en común.

Otro rasgo transversal a todas las experiencias puede verse en la generación de un conocimiento hibridizado entre la arquitectura, las artes plásticas, visuales y audiovisuales como resultado de una permanente migración. En este sentido, las obras resultantes salen de la convención de la representación arquitectónica tradicional. Una obra de arquitectura colaborativa no es fiel ni a las técnicas de representación, ni responde a un estilo puro. Por ejemplo, en el caso de TURBA, la estética del mapa es resultado de un consenso y responde a una estrategia comunicativa que busca impactar al interior de su comunidad. Saliendo de las convenciones de los mapas oficiales. En GAYA como en Pequeños Arquitectos ocurre algo similar. Las obras se alejan de la representación tradicional de la arquitectura para acercarse a las artes plásticas.

Para el caso de Ciudad Invisible, la situación se radicaliza porque la percepción táctil, auditiva y olfativa del espacio condujo al uso de herramientas, técnicas y materiales distintos a los que comúnmente se usan para representar la arquitectura.

Todas estas experiencias se contraponen a la idea totalizadora que subyace en el conocimiento disciplinario y en sus discursos institucionalizados. La arquitectura colaborativa entonces se construye desde la diversidad y busca fortalecer en cada caso identidades situadas. En este sentido, la identidad se activa en el momento en que un grupo social recupera, ante diversas circunstancias, la capacidad de "auto-identificarse" y "autocomprenderse". Sobre esto, Brubaker y Cooper (2001) dicen

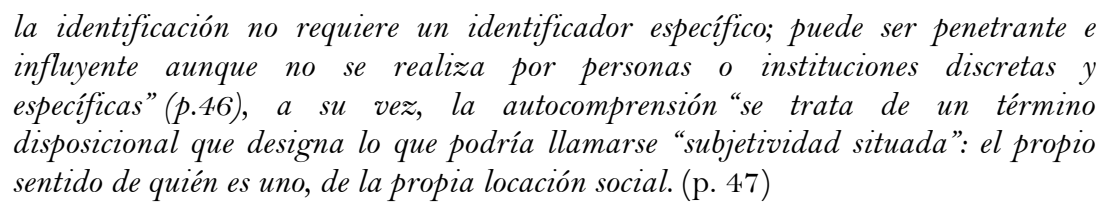

Para concluir amplío la escala del problema. La ciudad capitalista y su constante violación de derechos ciudadanos. Dejando como huella la enorme cantidad de 
comunidades vulnerables. Por ello y considerando los casos analizados, la formación en temas vinculados a la arquitectura y al urbanismo es fundamental para fortalecer la autogestión y participación comunitaria en instancias de mayor escala. Este enfoque alternativo al que llamo "arquitectura colaborativa" por encontrarse en vinculación con prácticas artísticas colaborativas, engendraron nuevas formas discursivas de la arquitectura. Otorgando un nuevo rol de las comunidades implicadas en la producción y representación territorial. Pudiendo generar otras instancias y replicarse a otros grupos con similares características o no.

Como plantee en el inicio del artículo, no pretendo develar algo que no esté practicándose. Afortunadamente tanto en la escena local como la internacional existen ejemplos que hacen aportes significativos en relación a este tema. Considero que es fundamental producir intercambios y acciones que permitan interpelar la frontera entre lo que es y no es la arquitectura. Queda mucho por pensar y hacer.

\section{Referencias}

Bourriaud, N. (2007). Estética relacional. Buenos Aires: Editorial Adriana Hidalgo.

Brubaker, R. y Cooper, F. (2001). Más allá de la identidad. Apuntes de Investigación del CECYP, 7, 30-67.

Claramonte, J. (2012). Modos de hacer. Arte crítico, esfera pública y acción directa. Salamanca: Universidad de Salamanca Ediciones.

Del Río Almagro, A. y Collados Alcalde, A. (2013). Modos y grados de relación e implicación en las prácticas artísticas colaborativas. Relaciones fluctuantes entre artistas y comunidades. Creatividad y Sociedad, 20(3), 1-24.

García Canclini, N. (1999). La globalización imaginada. Buenos Aires: Paidós

Garrido, A. (2009). El arte comunitario: origen y evolución de las prácticas artísticas colaborativas. Recuperado de http://www.ub.edu/hsctreballsocial/

Rocha, E. (2014). Hacia una geografía comunitaria. abordajes desde la cartografía social y sistemas de información geográfica. Pelotas: Universidad de Patagonia-CONICET.

\section{Breve CV del autor}

\section{Javier Samaniego}

Artista, arquitecto y educador popular. Se desempeña como docente en las materias Planificación Territorial y Teoría (Facultad de Arquitectura y Urbanismo-Universidad Nacional de La Plata). Es tesista de la Maestría de Paisaje Medioambiente y Ciudad (FAU - UNLP) y becario de la Academia Nacional de Bellas Artes. Ganó diferentes premios, becas y participó tanto en eventos artísticos (muestras, exposiciones, festivales) y académicos (congresos, encuentros, jornadas). Es Director de GAYA (Galería de Arte y Arquitectura) La Plata - Buenos Aires. Integra el colectivo de Arte y Cartografias TURBA V31 (Taller de Urbanismo Barrial Villa 31, CABA). Es devoto e investigador del Mito de Hígado García. ORCID ID: orcid.org/00o0-0003-2983-9160. Email: javiersamaniegog@gmail.com 